\title{
PROPERTIES OF ANTIMONY SELENIDE NANO CRYSTALLINE THIN FILMS
}

\author{
Hemlata Panwar, Vivek Bhojak, Swati Arora \\ E-Mail Id: hemlatap0@gmail.com \\ Department of Electronics \& Communication Engineering, Anand International College of Engineering \\ Jaipur, India
}

\begin{abstract}
Antimony Selenide has been saved on ITO substrate under the streamlined conditions utilizing vacuum covering unit with affidavit pace of $10 \mathrm{~A} \% \mathrm{sec}$. It has discovered that slender film had adherence and uniform to the substrate and no pores has been found. It was seen that optical band hole diminishes exponentially with Builds the Photon Vitality (Eg) It is additionally seen that assimilation coefficient in Optical Band Hole diminishes exponentially with expands wavelength of Photon $(\lambda \mathrm{nm})$. Arranged slender movies saved on appropriately cleaned substrate at various stoichiometry proportion $(75: 25,50: 50,20: 80)$ at consistent thickness of $200 \mathrm{~nm}$.Nano Crystalline flimsy movies were kept at without strengthened, toughened at $2000 \mathrm{C}$ to analyze the impact of tempering in recrystallization.The crystalline size has been resolved utilizing Scherer's recipe increments in range $76-94 \mathrm{~nm}$ with increment in strengthening temperature. At without toughening arranged slim movies were discovered indistinct in nature. Tests were considered by X-beam diffraction (XRD) and checking electron microscopy (SEM) to get exhaustive and predictable microstructural data. UV-VIS NIR Spectrometer is utilized to quantify Optical properties of slim films.X-Beam Photograph Electron Spectroscopy (XPS) was utilized to gauge the essential piece of dainty movies. A picosecond laser siphon test framework was utilized to examine the stage change time among indistinct and crystalline states, by estimating the reflectivity of the material. Raman Spectra Examination strategy used to watch and investigation optical spectra to analyze the surface morphology of slight movies.
\end{abstract}

\section{INTRODUCTION}

Se-Sb parallel chalcogenide Thin Films Due to the huge assimilation coefficient and compositional reliance of retention, appropriate for Solid state memory gadgets like Solar cells, photoconductors, Alloys, glass generation and so on. Antimony selenide ( $\mathrm{Sb} 2 \mathrm{Se} 3$ ) is a mindful semiconductor material because of its exchanging, photovoltaic, color blended sun based cells application. The two Materials have an aberrant vitality band hole shifts between $2.50 \mathrm{eV}$ to $1.753 \mathrm{eV}$ [1].

Writing indicates such a large number of strategies for testimony of flimsy movies, for example, warm dissipation statement, sub-atomic bar epitaxial, organ-metallic, substance vapour affidavit, arrangement development and so forth. The decision of the statement technique might be founded on nature of the movies required for explicit applications. Stoichiometry proportion of $\mathrm{Se}(1-\mathrm{x}) \mathrm{Sbx}$ Thin Films with $\mathrm{x}=(0.25,0.50$, 0.80) of various piece of $\mathrm{Se} 75-\mathrm{Sb} 25, \quad \mathrm{Se} 50-\mathrm{Sb} 50, \mathrm{Se} 20-\mathrm{Sb} 80$ kept onto an ITO substrate by warm vanishing technique under the vacuum of around 10-5torr by the beds utilizing vacuum covering unit.

Writing demonstrates that $\mathrm{Sb} 2 \mathrm{Se} 3$ utilizes in memory gadgets. In memory gadgets Phase change memory because of read and compose with quick speed, high thickness storage capacity and perfect with complementary metaloxide semiconductor (CMOS), which viewed as the most encouraging elective blaze memory, turns into the standard of the up and coming age of non - unpredictable capacity innovation. So as to tackle the issues of enormous activity present and warm impedance, the entire advancement pattern of PCM is 1 and the threedimensional bearing to the nanometre scale [2].

Optical Band Gap Energy of antimony selenide (Sb2Se3) semiconductor movies ingests low vitality light in a noticeable and close infrared locale. The utilization of Sb2Se3 semiconductor as a slender film was of essential enthusiasm to improve proficiency and sound qualities in sun powered cells. The optical stockpiling dependent on the indistinct crystalline stage change has been contemplated [3].

\section{EXPERIMENTAL DETAILS}

\subsection{Materials and Methods}

Selenium (Se) was bought from Koch-Light research centers LTD, Colebrook Berks England. Antimony (Sb) was bought from Sigma Aldrich research centers. Both the material have (99.99\%) immaculateness as powder structure. For statement the Se-Sb slight movies on Glass substrate the powder was changed over into little beds with various stoichiometry proportion.

\subsection{Synthesis and Characterization}

The cleaning of the substrate is huge for the statement of meager movies. ITO slides accessible economically with the size of $75 \mathrm{~mm} \times 25 \mathrm{~mm} \times 2 \mathrm{~mm}$ were washed by cleanser arrangement and consequently held back in hot chromic corrosive and after that cleaned with deionized water pursued by flushing in $\mathrm{CH} 32 \mathrm{CO}$. At last, the substrates were ultrasonically cleaned for $10 \mathrm{~min}$ with deionized water and cleaned with $\mathrm{CH} 32 \mathrm{CO}$ then put away in a hot stove. Selenium (Se) was bought from Koch-Light research facilities LTD, Colebrook Berks England. Antimony ( $\mathrm{Sb}$ ) was bought from Sigma Aldrich research centers. Both the material have (99.99\%) immaculateness as powder structure. For testimony the Se:Sb flimsy movies on substrate the powder was changed over into little beds with various stoichiometry proportion The careful extents of high virtue $(99.999 \%)$ 
$\mathrm{Se}$ and $\mathrm{Sb}$ components powder as per their nuclear rates were gauged utilizing an electronic equalization (LIBROR, - 120) with minimal tally of 10-4 gm and made the beds of Se:Sb Nano crystalline slim movies with various stoichiometry proportion [4]. The materials as a bed structure were set in high resistive tungsten pontoon which was warmed by implication by going high current through the terminals. The temperature of the heater was raised gradually at a pace of $3-4^{\circ} \mathrm{C} /$ minute. The Deposition rate was kept up at $4.4-7.6 \AA /$ sec. The pace of thickness and vanishing pace of the as-kept movies was checked utilizing quartz gem screen which work as sensor. To accomplish the metastable harmony the saved slight movies were kept inside the vacuum affidavit chamber for 2-3 hours.

\section{RESULTS AND DISCUSSION}

\subsection{Optical Band gap}

Optical properties of the prepared thin films were studied by measuring the optical absorption spectra using by an ultra violet - visible range UV-VIS spectrometer (Shimadzu Corporation make Probe 1800) [5]. The output absorbance can be recorded and printed out. The optical absorption of prepared Se: Sb thin films shown in Fig. 3.1 for different Stoichiometry ratio of $\mathrm{Se}: \mathrm{Sb}$ at different annealing temperature. It has been observed that absorption coefficient in Optical Band Gap decreases exponentially with increases wavelength of Photon $(\lambda \mathrm{nm})$. The absorption edge of Nano Crystalline thin film is located in visible range i.e. from $580 \mathrm{~nm}$ to $620 \mathrm{~nm}$. It may be due to an increase in energy density because of the higher concentration of Stoichiometry ratio of Se: Sb was further increases with annealing temperature. The band gap of the Se:Sb prepared thin film was calculated using Tauc's relation [6] shown in Fig. 2. and Table I for different stoichiometry ratio at different annealing temperature which shows that optical band gap decreases exponentially with Increases the Photon Energy (Eg). Absorption is defined as $\log 10$ (I0/I), where I0 is the incident wavelength intensity and I is the transmitted wavelength intensity. So absorption coefficient $(\alpha)$ can be written as

$$
\alpha=1 / \mathrm{d} \log 10(\mathrm{I} 0 / \mathrm{I})
$$

Where $\mathrm{d}$ is the absorption path length $[4,5]$.The absorption coefficient $(\alpha)$ of the thin films was calculated by the absorbance data at different photon energies, hv and different temperature.
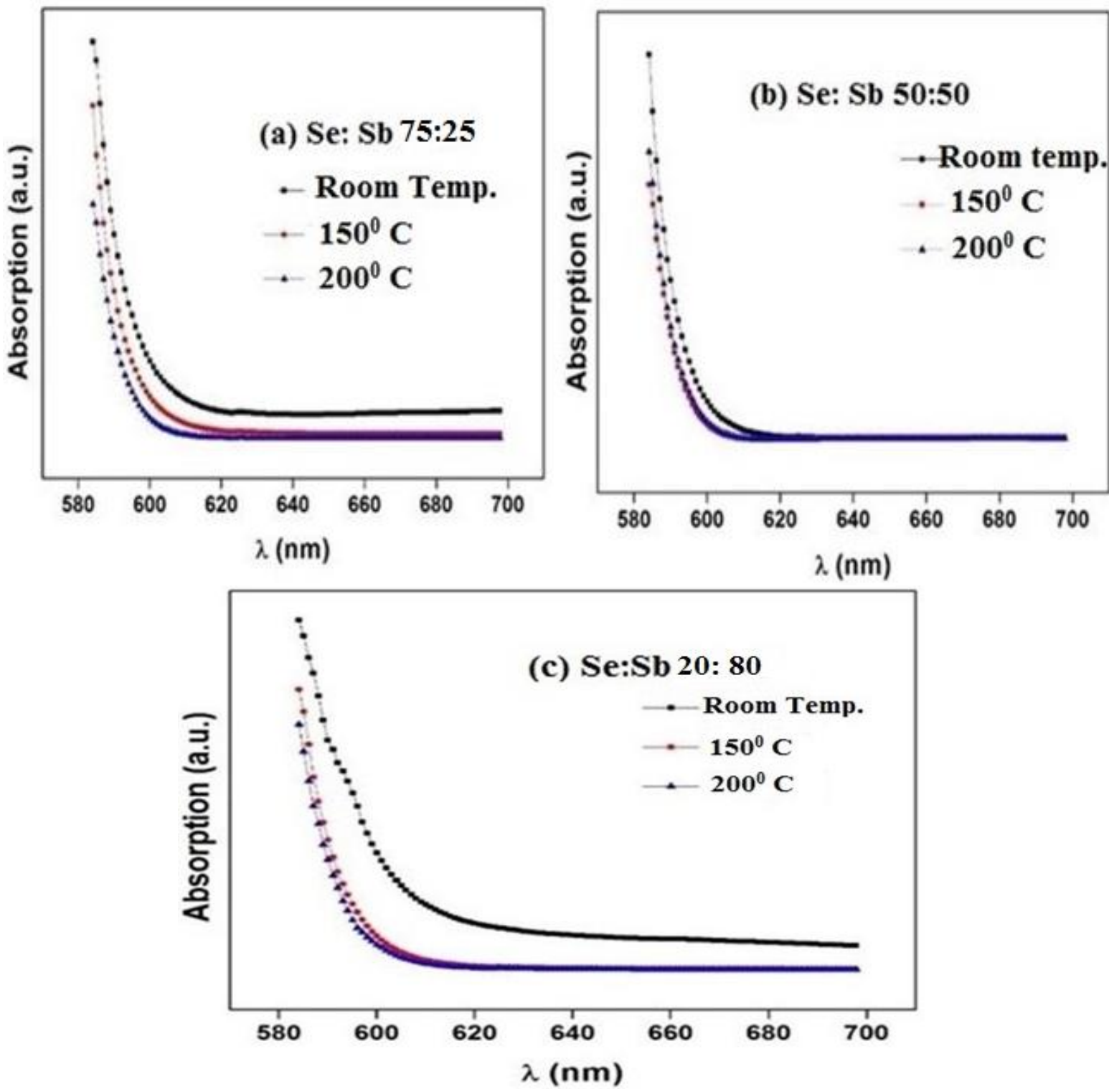

Fig. 3.1(a) Optical absorption curves for Se:Sb thin films (a) 75:25 (b) 50:50 (c) 20:80

DOI Number: https://doi.org/10.30780/specialissue-ICRDET-2019/005

Paper Id: IJTRS-ICRDET-005 
ICRDET-2019, September 14-15, 2019, AICE, Jaipur, India

International Journal of Technical Research \& Science (Special Issue) ISSN No.:2454-2024 (online)
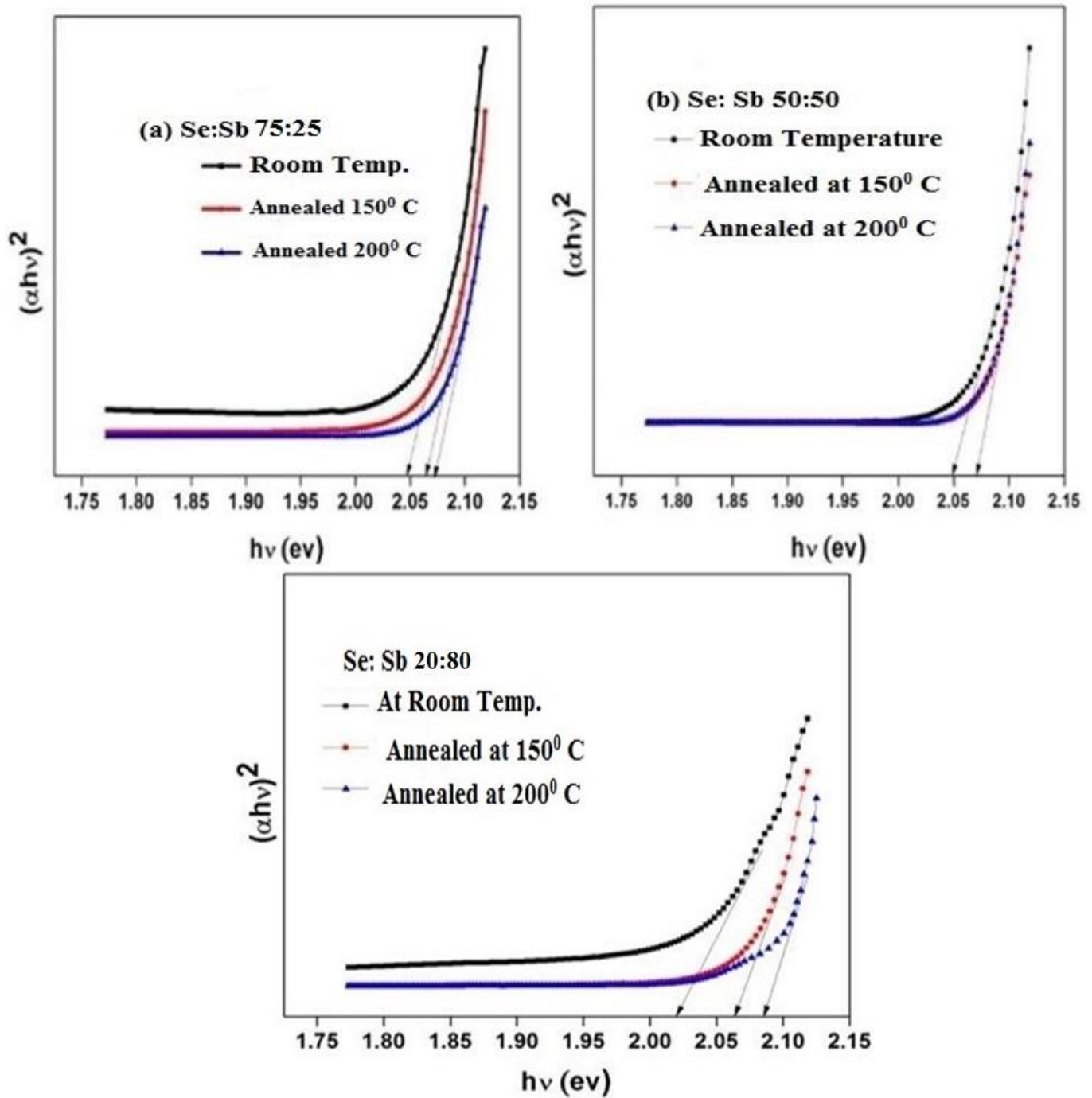

Fig. 3.1(b) (ahv)2 vs hv (eV) Curves for different Thickness Ratio of Se:Sb Thin Films (a) 75:25 (b) 50:50 (c) 20:80

Table 3.1 Band Gap Calculated Values of Se: Sb for Different Stoichiometry Ratio at Various Temperature

\begin{tabular}{|c|c|c|c|}
\hline \multirow{2}{*}{ Thickness Ratio } & \multicolumn{3}{|c|}{ Optical Band gap(eV) } \\
\cline { 2 - 4 } & RT & $\mathbf{1 5 0 0 C}$ & 200oC \\
\hline $25: 75$ & 2.04 & 2.06 & 2.07 \\
\hline $50: 50$ & 2.04 & 2.07 & 2.07 \\
\hline $75: 25$ & 2.01 & 2.06 & 2.08 \\
\hline
\end{tabular}

3.2 X-ray diffraction analysis

The structure of the film and the phase composition has been studied by $\mathrm{X}$ ray diffraction analysis [5]. To identify the structure of the deposited thin film. The XRD patterns of the deposited and annealed Se (1-x) Sbx thin films shown in Fig.3. The XRD spectrum exhibits the multiple characteristics peaks at 20. Further, no additional peak was observed for the annealed sample as compared to the as-deposited thin films, that indicating that no new interfacial phase was formed after annealing. It has also been observed that peak intensity increases with increase the annealing temperature. It is also observed that No crystallize phase appeared in without annealed thin films at room temperature which show amorphous nature of thin films. But after annealing crystallization phase appeared as the peak of Antimony Tri Selenide (Sb2Se3) at 200o C and grain particles arrange in more crystalline form on surface of substrate which shows polycrystalline nature of thin films.

Fig 3 (a) shows the excellent peaks (211) (330) (311) (160) (621) (720) (370). It forms orthorhombic ${ }^{\circ}$ structure with value of $\mathrm{a}=11.63 \mathrm{~A}{ }^{\circ}, \mathrm{b}=11.78 \mathrm{~A}^{\circ}, \mathrm{c}=3.98 \mathrm{~A} \mathrm{C}(4,5,6)^{\circ}$ were obtained and Fig 3 (b) shows after

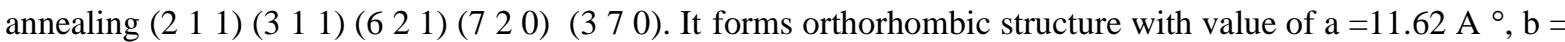
$11.77 \mathrm{~A}{ }^{\circ}, \mathrm{c}=3.96 \mathrm{~A}^{\circ}$ in the powder Xray diffraction studies. The peaks were compared with JCPDS diffraction patterns from the JCPDS Data File No [(72-1184)]. The formation of hexagonal phase observed the peaks corresponding to $\mathrm{Se}: \mathrm{Sb}$ were indexed according to hexagonal structure.

DOI Number: https://doi.org/10.30780/specialissue-ICRDET-2019/005

pg. 28 
ICRDET-2019, September 14-15, 2019, AICE, Jaipur, India

International Journal of Technical Research \& Science (Special Issue) ISSN No.:2454-2024 (online)

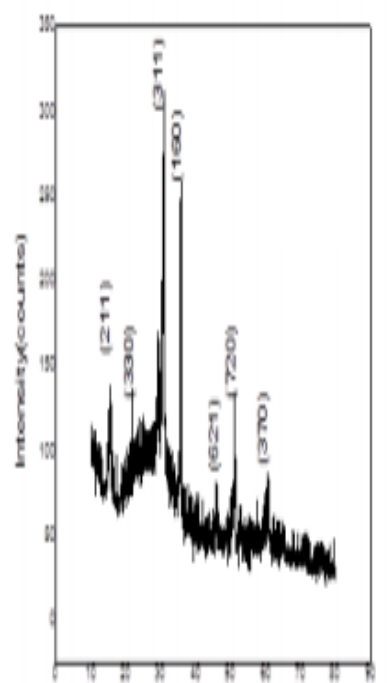

and

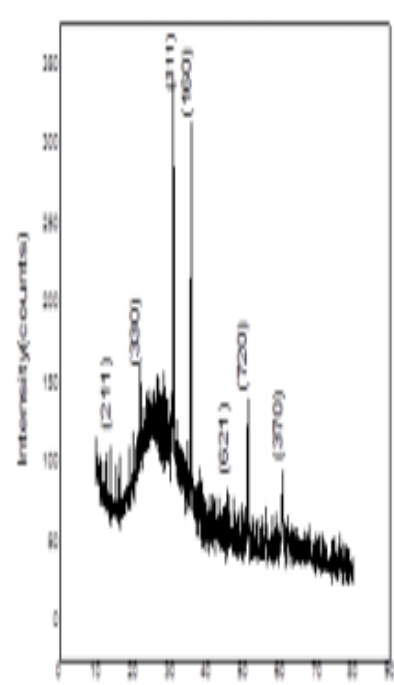

ididiaj

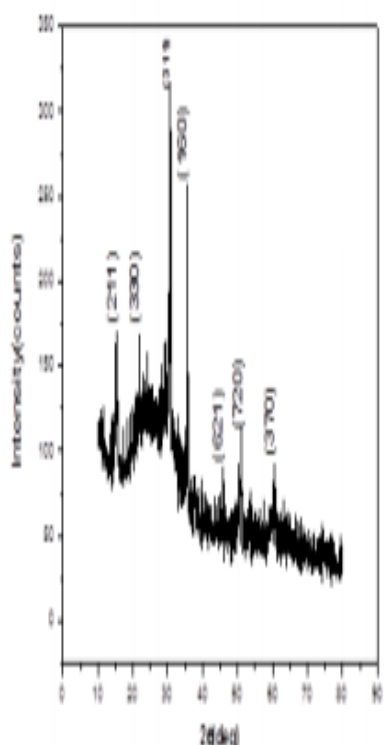

(itas)

Fig. 3.2(a) XRD patterns of the deposited Se: Sb thin films (a) 75:25 (b) 50:50 (c) 20:80
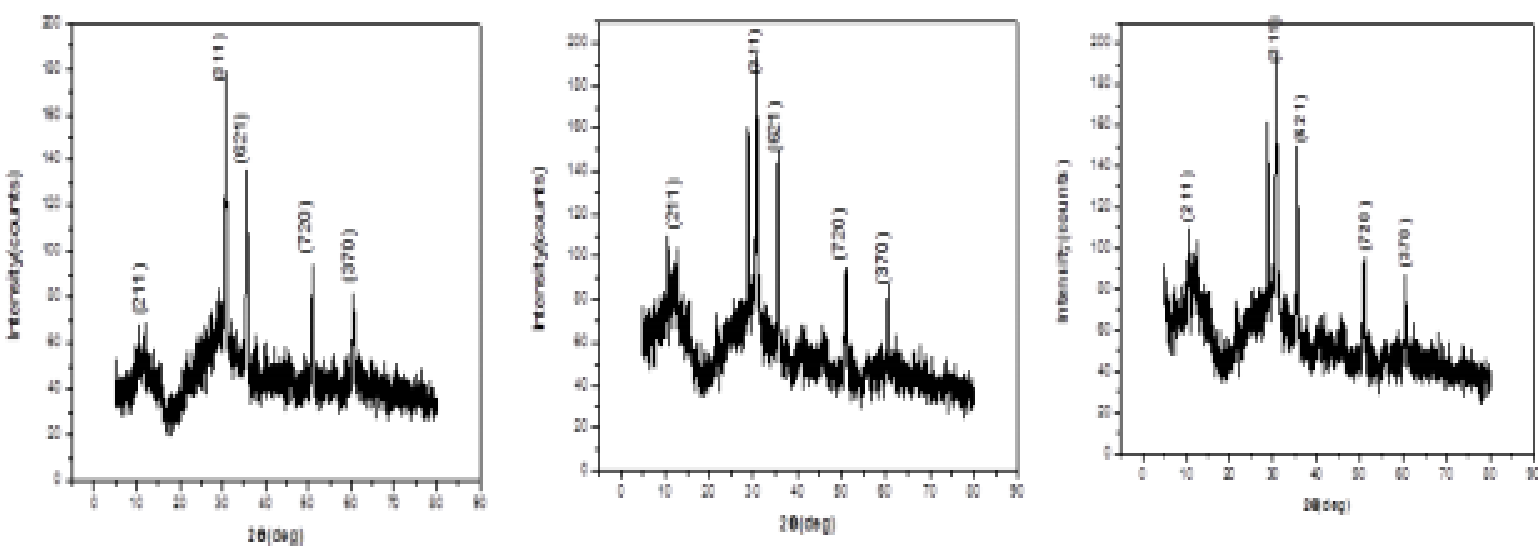

Fig. 3.2(b) XRD patterns of annealed Se: Sb thin films (a) 75:25 (b) 50:50 (c) 20:80 at 200o C

Crystallite size (D) was calculated using Debye-Scherrer's formula [3]

$$
\mathrm{D}=\frac{0.9 * \lambda}{\beta * \cos \theta}
$$

Where $\mathrm{D}$ is the crystallite size, $\lambda$ is the wavelength of the $\mathrm{k} \alpha$ line, $\beta$ is the full width at half maxima (FWHM) in radians and $\theta$ is the Bragg's angle. The crystallite grain size increased from $76-94 \mathrm{~nm}$ as the annealing temperature increased [4].

\subsection{SEM analysis}

The surface morphology of thin films was analyzed by Nova Nano SEM 450 make scanning electron microscopy (SEM) $[4,6]$.
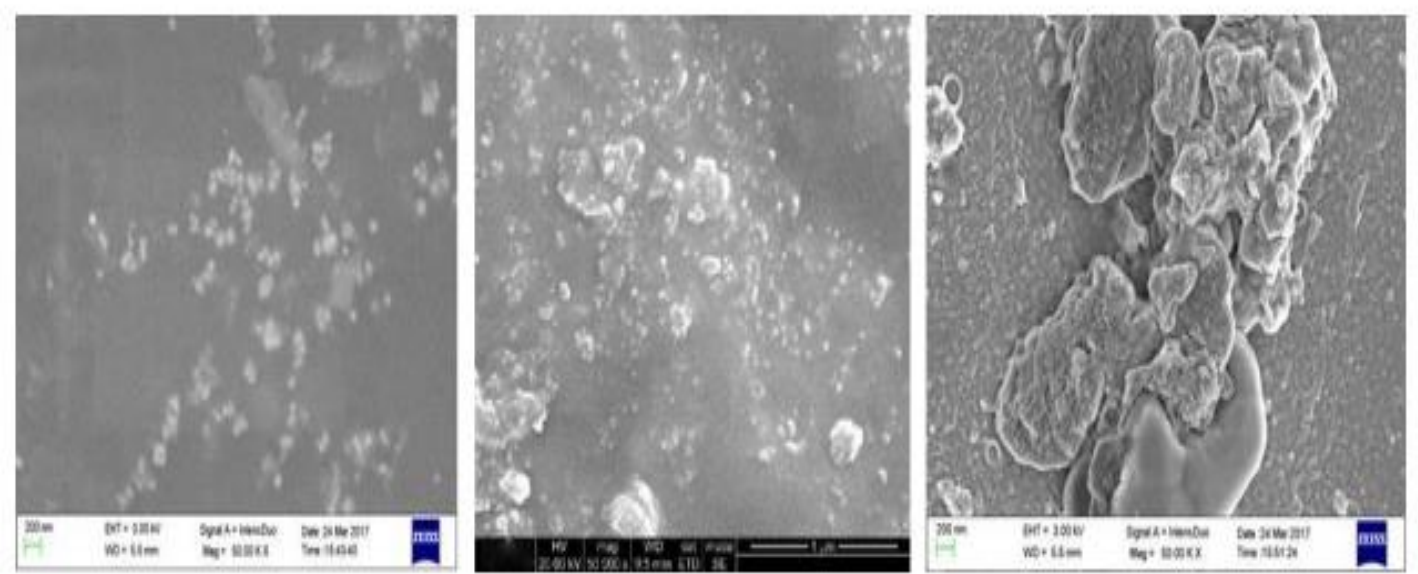

Fig. 3.3 (a) SEM Image of Deposited Se: Sb thin films (a) 75:25 (b) 50:50 (c) 20:80

DOI Number: https://doi.org/10.30780/specialissue-ICRDET-2019/005

Paper Id: IJTRS-ICRDET-005

pg. 29 @ 2017, IJTRS All Right Reserved, www.ijtrs.com 

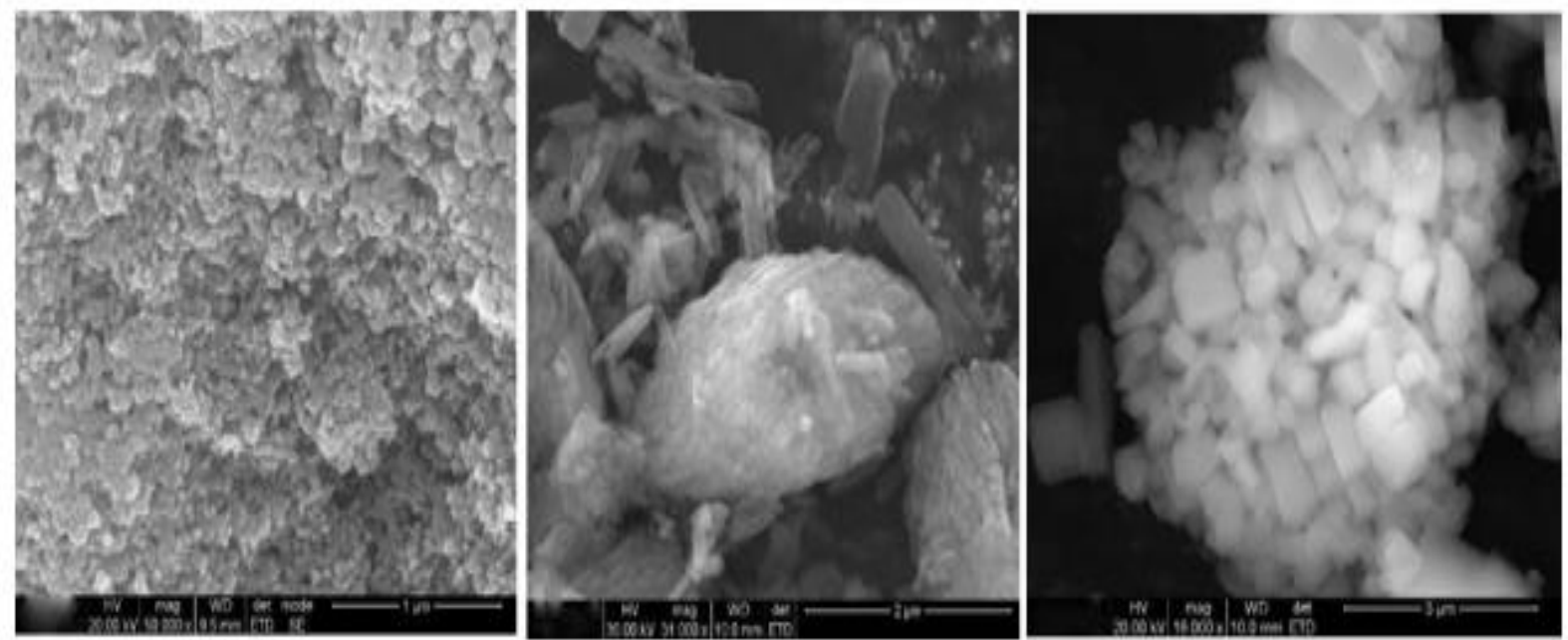

Fig. 3.3(b) SEM Image of Annealed Se: Sb Thin Films (a) 75:25 (b) 50:50 (c) 20:80 at 200 C

The SEM images of Se (1-x) Sb x thin films at magnification 10 um shown in Fig. 4. (a) Without annealed thin films (b) Annealed thin films. Without annealed thin films shows amorphous nature of thin films which indicate that granular particles grow on substrate as spherical granular manner which show smoothness of the surface with polycrystalline nature of thin films. At without annealing granular particle connected to each other by co-valent bonding between interatomic particles due to this surface shows the smoothness of thin films. But After annealing the surface morphology of thin films changed considerably which shows that big cluster and uneven lumps have appeared in spherical shape and are distributed on the entire surface. These clusters may be formed due to the agglomeration of particles during to heat treatment. After annealing the co-valent bonding between interatomic particles respites and Selenium ( $\mathrm{Se}$ ) deposited into Antimony ( $\mathrm{Sb}$ ) and make compound of Antimony Tri Selenide (Sb2Se3). After annealing thermal vibration between inter atomic molecules increases due to it there observed lamped gaps between granular particles. According to these SEM images we may conclude that as-deposited thin films have partially amorphous structure while after annealing the thin films become polycrystalline in nature.
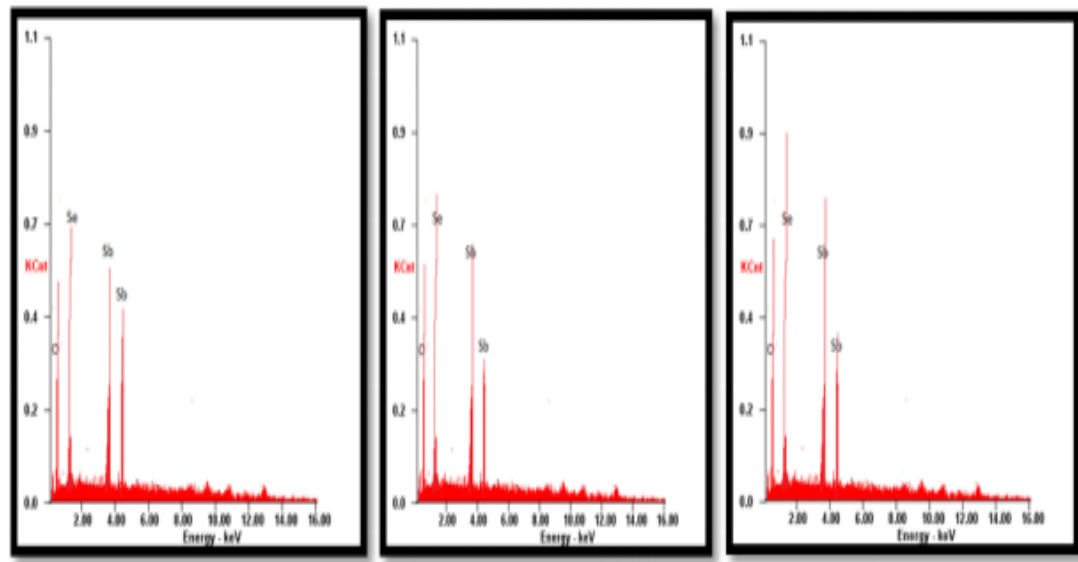

Fig. 3.3(c) Shows the EDAX Spectrum Recorded in the Binding Energy Region of 0-16 keV Which Shows the Presence of Antimony and Selenium in Thin Films

\subsection{Photoluminescence Spectrum}
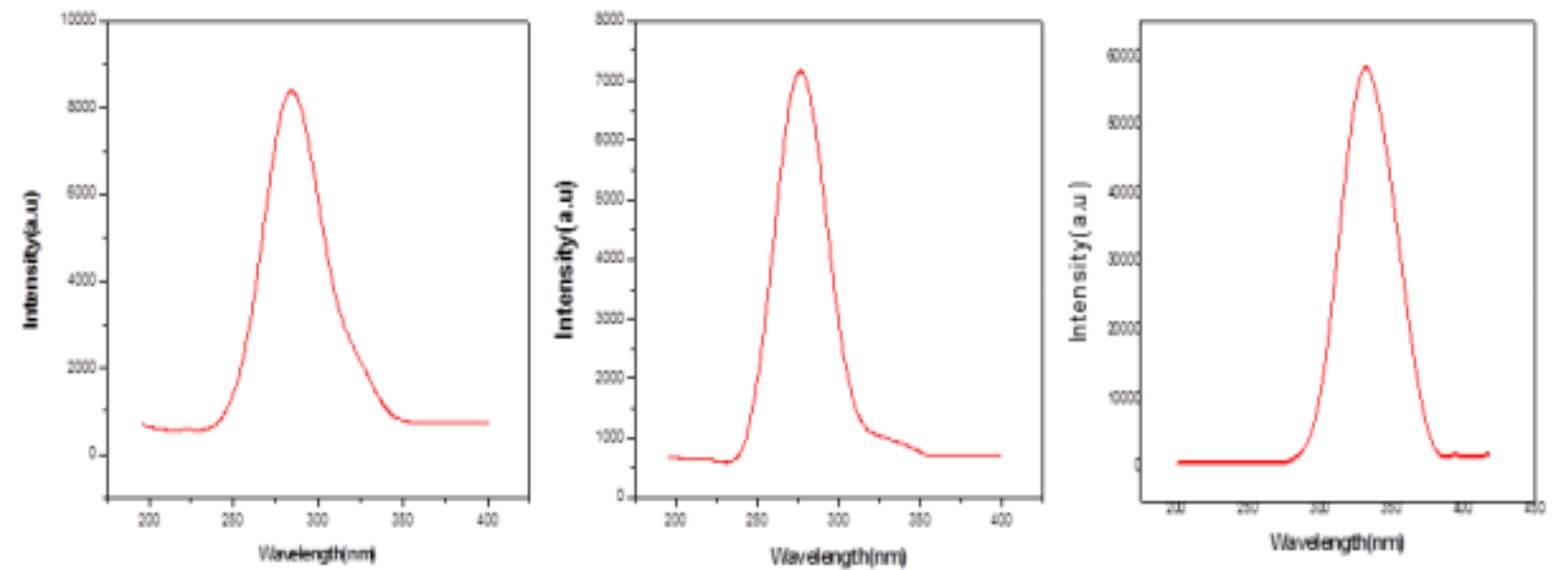

Fig. 3.4 Photoluminescence Spectra for Se:Sb with Ratio of 75:25 and 50:50 Annealed at $200^{\circ} \mathrm{C}$

DOI Number: https://doi.org/10.30780/specialissue-ICRDET-2019/005

Paper Id: IJTRS-ICRDET-005

pg. 30

@ 2017, IJTRS All Right Reserved, www.ijtrs.com 
The photoluminescence spectra of Se (1-x) Sbx thin films have been shown in Figure.6. The calculated band gap by the Photoluminescence spectrum ranges from 4.37 to $3.7 \mathrm{eV}$ as the time variation increases. The decrease in the band gap is observed as the time variation increases in Photoluminescence spectrum. As the time variation increases, the band gap of Antimony Selenide decreases.

\subsection{Raman Spectra Analysis}

Raman spectrum analysis was done by Micro Raman Spectrometer manufactured by Renishaw private ltd., Used two types of laser- $514 \mathrm{~nm}$ and $785 \mathrm{~nm}$ with different magnification lances to measure the spectrum analysis. Raman Spectra Analysis technique used to observe vibration, rotational, and other low-frequency modes in thin films. Optical characterization involved Raman spectroscopy and absorption spectra of Se: Sb thin films [6]. The Raman spectrum of a $17 \mathrm{~nm}$ thick. Se: Sb thin films of ratio 50:50 at without annealing shown in Fig 7.(a), where the excitation wavelength was $532 \mathrm{~nm}$. It shows the signature peaks corresponding to both the in-plane (E) and outof plane (A) modes that are consistent with those reported for flakes of $\gamma$-wave intensity. Comparing between without annealed and annealed results for the bulk and crystalline thin films in Fig.7, the peaks identify at about $117 \mathrm{~cm}-1,175 \mathrm{~cm}-1$, and $205 \mathrm{~cm}-1$ with A1, E2g, and A1 (LO) Raman modes, respectively. The peak at 205 $\mathrm{cm}-1$ is strongest in films that are at about $6 \mathrm{~nm}$ to $9 \mathrm{~nm}$ thick layers and then attenuates with increase in thickness. The peak at around $155 \mathrm{~cm}-1$ is distinctive for the $\gamma$ phase of Se: Sb and is related to the zone centre mode of the crystal. The Raman spectrum of the thin films which was $15 \mathrm{~nm}$ thick, shown in Fig. 7. (b). which shows that the peak phase height increases after annealing of thin films.
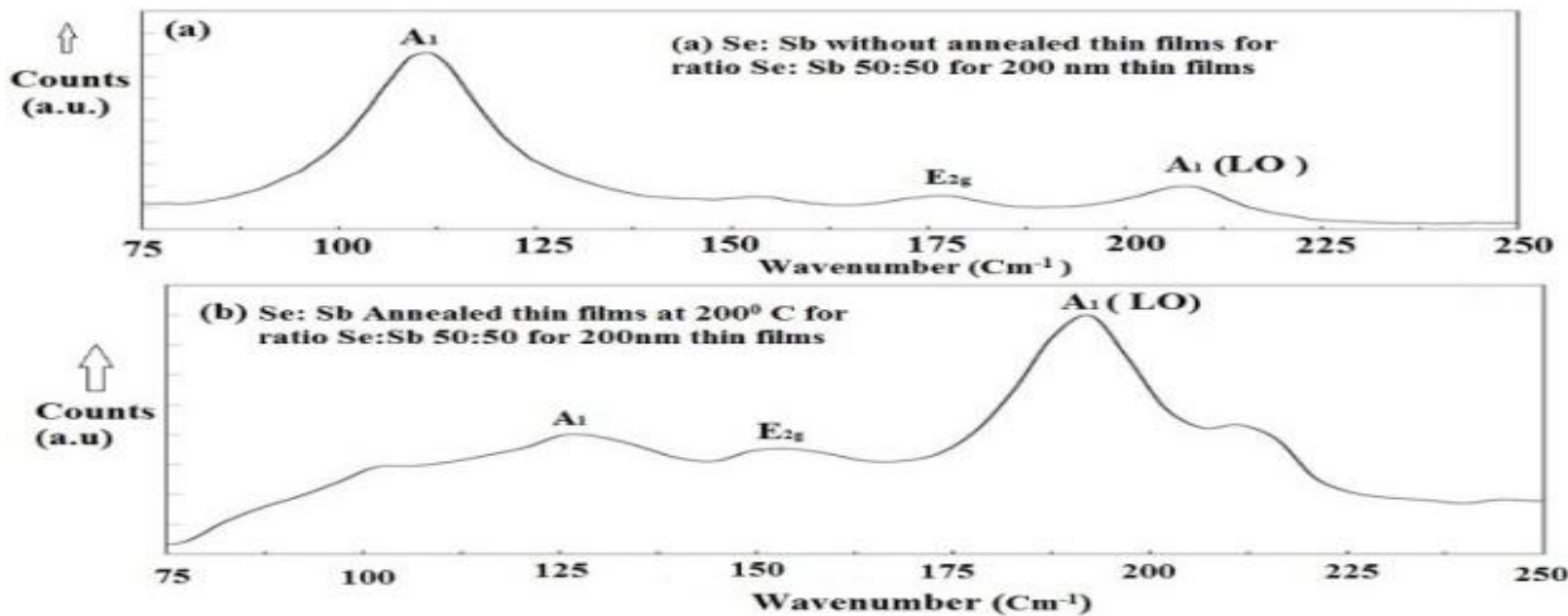

Fig. 3.5 Raman Spectrograph of Se: Sb thin films of 50:50 at (a) Without annealed (b) Annealed at $200{ }^{\circ} \mathrm{C}$

\subsection{XPS Analysis of Thin Films}

X- Ray Photo-Electron Spectroscopy (XPS) manufactured by Auger Electron Spectroscopy (AES) module and C60 sputter Gun model PHI 5000 versa probe II used to measure the element composition [7]. X-ray photoelectron spectroscopy (XPS) is a surface-sensitive quantitative spectroscopic technique that measures the elemental composition at the parts per thousand range, chemical state and electronic state of the elements that exist within a material. XPS spectra are obtained by irradiating a material with a beam of X-rays while simultaneously measuring the kinetic energy and number of electrons that escape from the top 0 to $10 \mathrm{~nm}$ of the material being analysed. XPS requires high vacuum ( $\mathrm{P} \sim 10-8$ millibar).
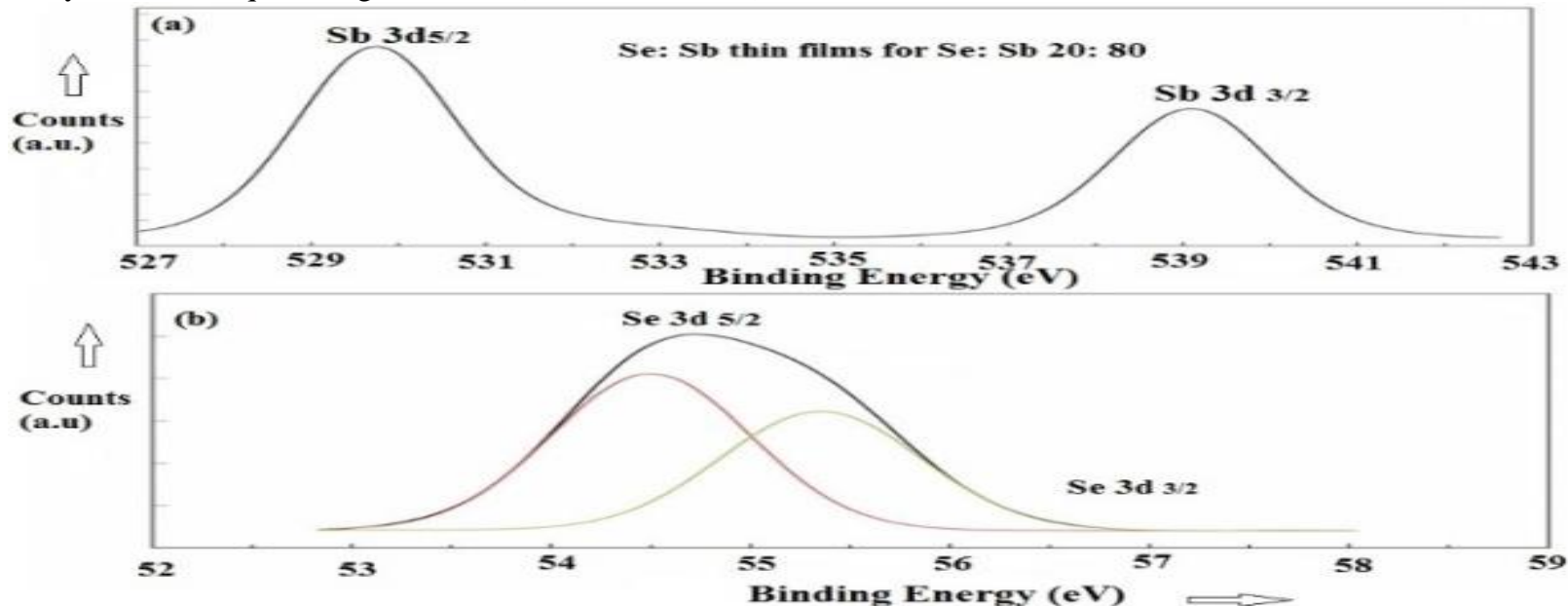

Fig. 3.6(a) X-Ray Photoelectron Spectroscopy (XPS) profile of (a) Sb (b) Se for ratio of Se: Sb 20:80

DOI Number: https://doi.org/10.30780/specialissue-ICRDET-2019/005

Paper Id: IJTRS-ICRDET-005

pg. 31

@ 2017, IJTRS All Right Reserved, www.ijtrs.com 
The XPS binding energy of Se: Sb thin films for stoichiometric ratio Se: Sb 20:80 shown in Fig. 6. The binding energies of $\mathrm{Sb} 3 \mathrm{~d} 5 / 2$ and $3 \mathrm{~d} 3 / 2$ are $529.83 \mathrm{eV}$ and $539.03 \mathrm{eV}$ as shown in Fig. 8. (a). Detailed spectral deconvolution of the Se $3 d$ high resolution XPS spectrum revealed that the binding energy of Se 3d5/2 and 3d3/2 are $54.43 \mathrm{eV}$ and $55.23 \mathrm{eV}$, respectively, which is in good agreement with the expected binding energy of Sb2Se3. The chemical composition of both of these films was examined by X-ray photoelectron spectroscopy (XPS). The signature binding energies of $\mathrm{Sb} 3 \mathrm{~d} 5 / 2$ and $\mathrm{Sb} 3 \mathrm{~d} 3 / 2$, shown in Fig. 9. For ratio of Se: Sb 75:25 correspond to $452.47 \mathrm{eV}$ and $444.88 \mathrm{eV}$, respectively. The Se $3 \mathrm{~d}$ profile is DE convoluted to show the two, closely spaced binding profiles of the Se $\mathrm{d} 5 / 2$ and $\mathrm{d} 3 / 2$ of binding energies of $53.88 \mathrm{eV}$ and $54.88 \mathrm{eV}$ shown in Fig.9. (b). The dip between the two peaks is an indication of oxidation of the films, which is not surprising since the samples were exposed to air for a short period of time before XPS analysis [8].

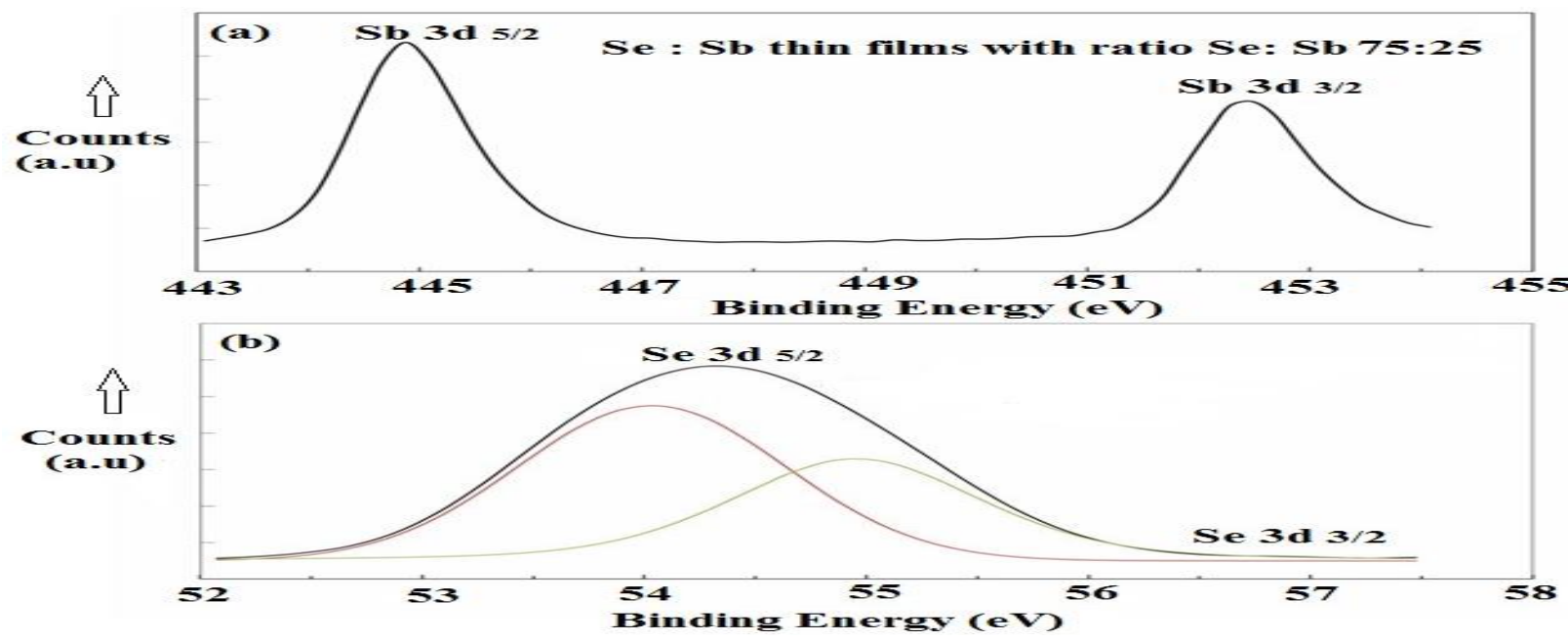

Fig. 3.6(b) X-Ray Photoelectron Spectroscopy (XPS) Profile of (a) Sb (b) Se for ratio of Se: Sb 75:25

\subsection{Phase Change Time Analysis}

A picosecond laser pump-probe system was used to investigate the phase-change time between amorphous and crystalline states, by measuring the reflectivity of the material [9]. The light source used for irradiating the samples was a frequency-doubled model-locked neodymium yttrium aluminium garnet laser operating at $532 \mathrm{~nm}$ wavelengths at pulse duration of 30ps. In the phase change, the electrical resistivity changes are accompanied by optical reflectivity. In this study, the switching speed of the phase-change materials was investigated by picosecond laser technology [10]. Since the reset operation needs more power and shorter time than the set one in the resistance switching process of PCM devices, the reset power and the set speed have been attracted more attention. The power consumption and operation speed of PCM (Pulse code modulation) are mainly determined by the reset and set processes, respectively. Fig 10. Shows the normalized reflectivity evolution of the Se: Sb thin films with (a) at without annealed thin films (b) annealed thin films at $200{ }^{\circ} \mathrm{C}$ at different stoichiometric ratio. In the picosecond laser test, the time of 0 is a reference for data recording, and it does not correspond to the time of phase transition. In present study the time interval from amorphous state to crystalline state studied which appears as abrupt change in phase of reflectivity $[11,12]$. The amorphous state time was $1.60 \mathrm{~ns}$ and $0.96 \mathrm{~ns}$, which corresponds to the irradiation fluencies of $15.5 \mathrm{~mJ} / \mathrm{cm} 2$ and $23.8 \mathrm{~mJ} / \mathrm{cm} 2$ respectively, as shown in Fig. 10. (a), the crystallization time was observed at $1.7 \mathrm{~ns}$ under the irradiation fluency of $6.34 \mathrm{~mJ} / \mathrm{cm} 2$. It has been reported that the crystallization state time was $1.7 \mathrm{~ns}$. So conclusion is that reflectivity change efficiency as a phase change time at without annealing, irradiation fluencies decreases and amorphous state time of peak changes increases from amorphous nature of thin films at without annealing to crystallize state of thin films after annealing[12]

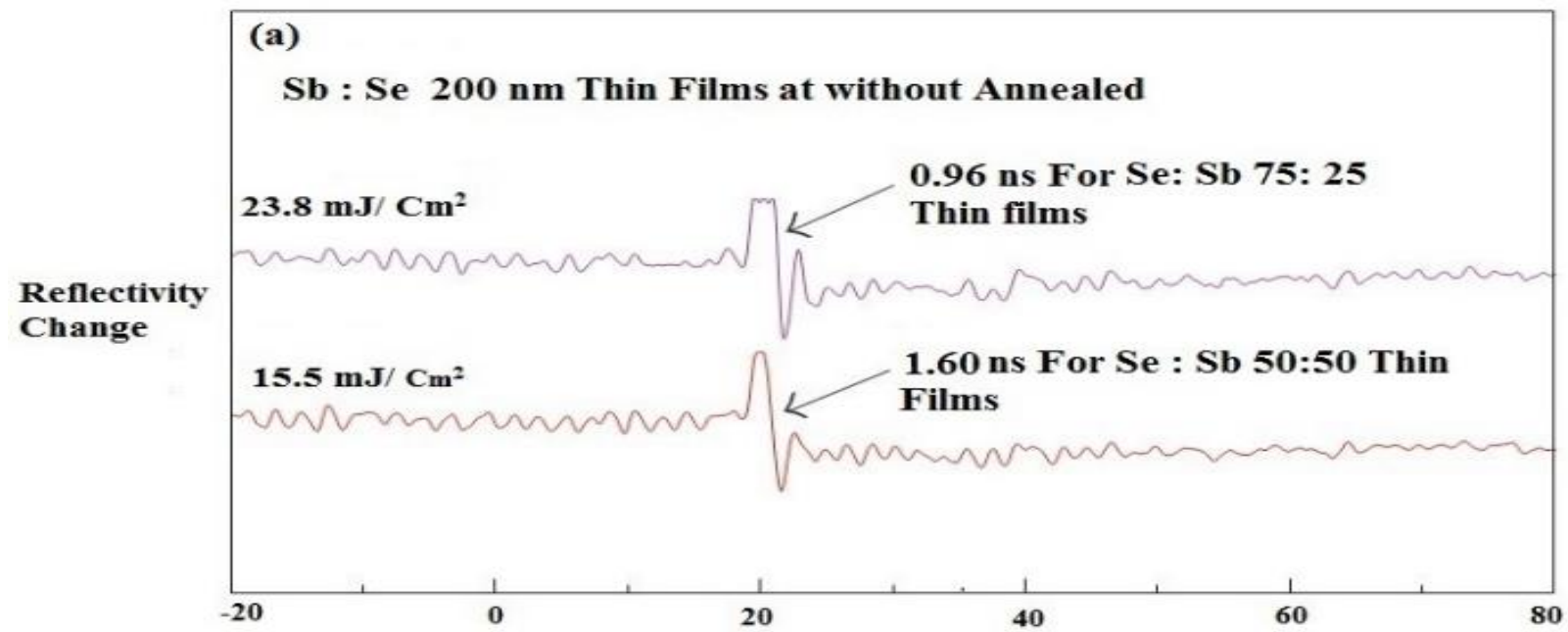

DOI Number: https://doi.org/10.30780/specialissue-ICRDET-2019/005

pg. 32 


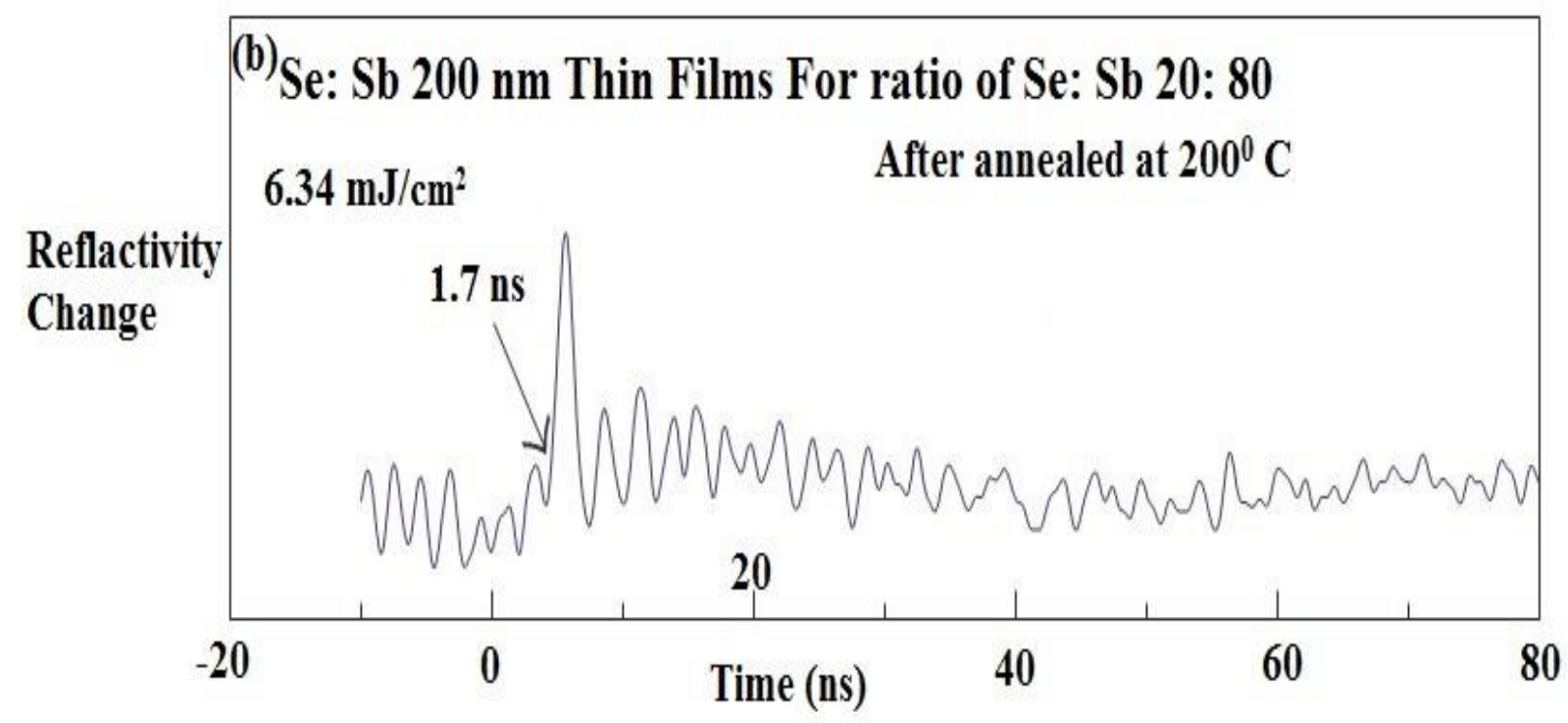

Fig. 3.7 Reflectivity Change Evolution of Se:Sb (a) Without Annealed 25:75 and 50:50 (b) Annealed at 200 ${ }^{\circ} \mathrm{C}$ of 20:80

\subsection{Structural Properties of the Thin Films}

To identify the structure of the deposited thin films, X-ray diffraction method is used. The XRD patterns of the deposited and annealed $\mathrm{Se}(1-\mathrm{x})$ Sbxthin films shown in Fig.6.The XRD spectrum exhibits the multiple characteristics peaks at $2 \theta$. Further, no additional peak was observed for the annealed sample as compared to the asdeposited thin films, that indicating that no new interfacial phase was formed after annealing. It has also been observed that peak intensity increases with increase the annealing temperature. It is also observed that No crystallize phase appeared in without annealed thin films at room temperature which show amorphous nature of thin films. But after annealing crystallization phase appeared as the peak of Antimony Tri Selenide ( $\mathrm{Sb} 2 \mathrm{Se} 3)$ at 150o $\mathrm{C}$ and 200o $\mathrm{C}$ and grain particles arrange in more crystalline form on surface of substrate which shows polycrystalline nature of thin films.

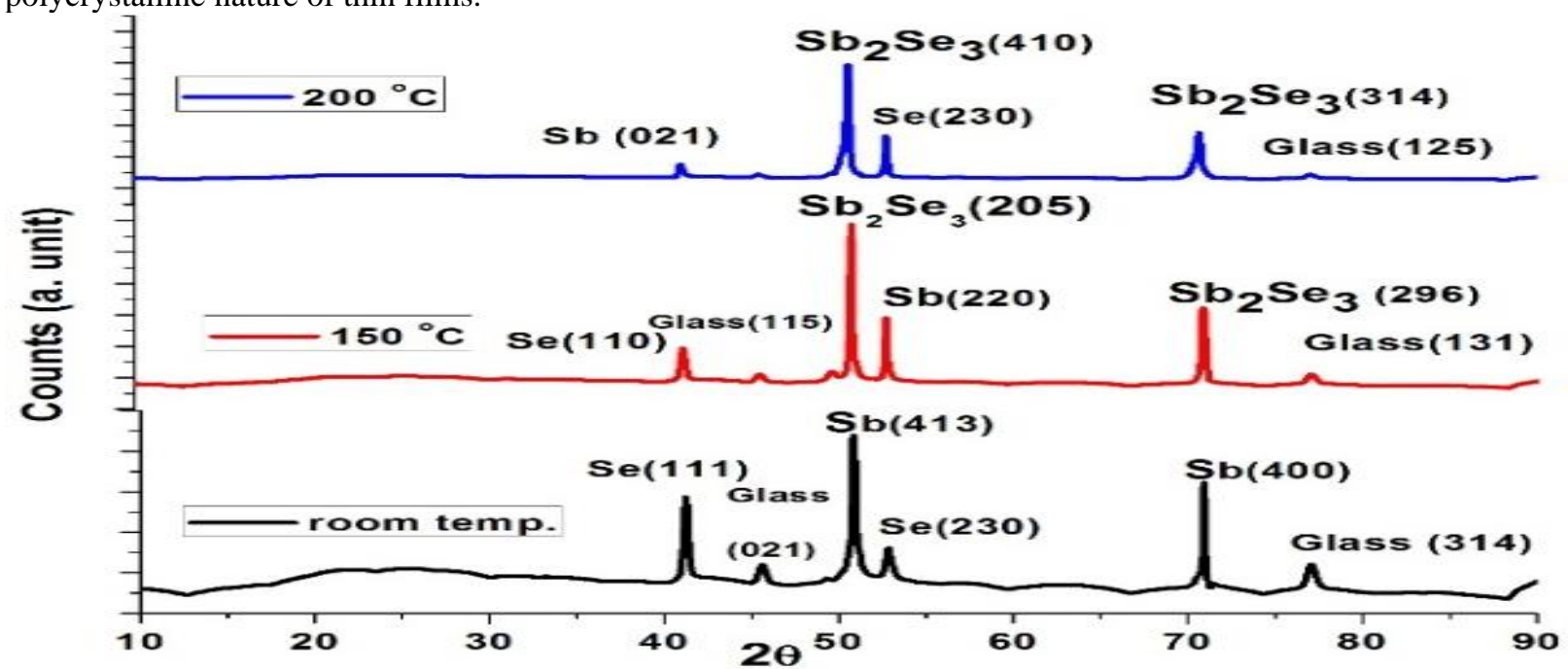

Fig. 3.8 Combined XRD Spectra of Thin Films for 200nm

\section{CONCLUSION}

Thin films of $\mathrm{Se}_{(1-\mathrm{x})} \mathrm{Sb}_{\mathrm{x}}$ of thickness $200 \mathrm{~nm}$ with different stoichiometry ratio were prepared on Glass substrate by thermal evaporation method under the vacuum of about $10^{-5}$ torr, using vacuum coating unit. A direct optical band gap between $2.04 \mathrm{eV}$ to $2.01 \mathrm{eV}$ is obtained at without annealing which shows that optical band gap decreases exponentially with Increases the Photon Energy $\left(\mathrm{E}_{\mathrm{g}}\right)$. It was also observed that absorption coefficient in Optical Band Gap decreases exponentially with increases wavelength of Photon $(\lambda \mathrm{nm})$. To find out the effect of recrystallization thin films of $\mathrm{Se}_{(1-x)} \mathrm{Sb}_{\mathrm{x}}$ were annealed at $150^{\circ} \mathrm{C}$ and $200^{\circ} \mathrm{C}$. The decrease in optical band gap with increase in $\mathrm{Sb}$ concentration may be due to the increase in the amount of disorder in the materials and increase in the density of defect states. Samples were studied by X-ray diffraction (XRD) and scanning electron microscopy (SEM) to obtain comprehensive and consistent micro structural information. Raman Spectra Analysis technique used to observe and analysis optical spectra to examine the surface morphology of thin films. Raman Spectra analysed at without annealed and Annealed at $200^{\circ} \mathrm{C}$ which shows that peak height increases after annealing. XRay Photo- Electron Spectroscopy (XPS) was used to measure the element composition of thin films. The 
molecular Binding energy calculated at different stoichiometry ratio of thin films. A picosecond laser pump-probe system was used to investigate the phase-change time between amorphous and crystalline states, by measuring the reflectivity of the material. The was that, reflectivity change efficiency as a phase change time at without annealing, irradiation fluencies decreases and amorphous state time of peak changes increases from amorphous nature of thin films at without annealing to crystallize state of thin films after annealing.

\section{REFERENCES}

[1] K. Zawawi, M. A. Mahdy, E. A. Sayad, "Influence of Film Thickness and Heat Treatment on the Physical Properties of Mn Doped Sb2Se3 Nano Crystalline Thin Films", Hindawi Journal of Nanomaterials, (2017).

[2] D.Zhang, Y. Hu, H.You, X. Zhu,Y.Sun, H. Zou, Y. Zheng, "High Reliability and Fast-Speed Phase-Change Memory Based on Sb70Se30/SiO2 Multilayer Thin Films", Hindawi Advances in Materials Science and Engineering, (2018).

[3] S.Thirumavalavan,K Mani,S Sagadevan, "Investigation of the Structural, Optical and Electrical Properties of Copper Selenide Thin Films", Materials Research, (2015).

[4] R H Bari, L APatil, "Synthesis and Characterization of Bismuth Selenide thin films by Chemical Bath Deposition Techniques", Indian Journal of Pure and applied Physics, (2010).

[5] V. Rajendran, R. Anitha, R. Dhanalakshmi, R Vijayalakshmi "Synthesis and characterization of Antimony Selenide Thin Film" International Journal of Nanomaterials in Renewable Energy Devices" (2018).

[6] R.Browning,N Kuperman, B Moon, RSolanki, "Atomic Layer Growth of InSe and Sb2Se3 Layered Semiconductors and Their Heterostructure", Journal of Electronics Materials,(2017).

[7] R.A. Chikwenze, P. A. Nwofe, P.E. Agbo, "AnnealingEffects and Film Thickness Dependence of Antimony Selenide Thin Films Grown By the Chemical Bath Deposition Method", IOSR Journal of Electronics and Communication Engineering, (2015).

[8] K Nagar, Amravati, "Deposition and Characterization of Antimony Selenide Thin Films", Metalogic in Science an International Refereed \& Indexed Quarterly Journal, (2012).

[9] Y. Li, Y. Zhou, J. Luo, W. Chen, B. Yang, X. Wen, S. Lu, C. Chen, K. Zeng, H. Son gab, J. Tang, "The effect of sodium on antimony selenide thin film solar cells" Royal Society of Chemistry, (2016).

[10] J.G. Garza, S. Shaji, A.M. Arato, E. P Tejerina, A. C. Rodriguez, T. K. Das Roy, B. Krishnan, "Chemically Deposited Silver Antimony Selenide Thin Films for Photovoltaic Applications", Materials Research Society, (2009).

[11] Swati Arora, Hemlata Panwar "Annealing Effects on Properties Nanocrystalline Thin Films" Journal of Nanoelectronics \& Optoelectronics, (2019).

[12] Swati Arora, Vivek Jaimini, Subodh Srivastava and Vijay, Y.K, Properties of nanostructure bismuth telluride thin films using thermal evaporation. Journal of Nanotechnology, (2017). 